\title{
ANÁLISIS DEL COMPORTAMIENTO BURSÁTIL DE LAS PRINCIPALES BOLSAS FINANCIERAS EN EL MUNDO USANDO EL ANÁLISIS MULTIVARIADO (ANÁLISIS DE COMPONENTES PRINCIPALES PCA) PARA EL PERIODO DE 2011 A 2014
}

\author{
Analysis of stock market behavior of the major financial exchanges worldwide using multivariate \\ analysis (principal component analysis PCA) for the period 2011 to 2014 \\ Christian Arturo Quiroga Juárez \\ Maestro en Administración. Universidad Politécnica del Bicentenario, Guanajuato - México, \\ cquirogaj@upbicentenario.edu.mx \\ Aglaé Villalobos Escobedo \\ Maestra en Administración. Universidad de Guanajuato. Guanajuato - México, licaglaeve@gmail.com
}

\section{Cómo citar/ How to cite}

Quiroga, C. y Villalobos, A. (2015). Análisis del comportamiento bursátil de las principales Bolsas Financieras en el mundo usando el análisis multivariado (análisis de componentes principales PCA) para el período 2011 a 2014. Revista CEA, 1(2), 25-36.

Recibido: 16 de abril de 2015

Aceptado: 2 de junio de 2015

\section{Resumen}

El objetivo de esta investigación es analizar el comportamiento bursátil de 27 bolsas financieras en el mundo, mediante el análisis de componentes principales PCA (por sus siglas en inglés). Esta técnica permite agrupar las variables correlacionadas entre sí separándolas de las que no, obteniendo un nuevo espacio reducido en donde se espera la formación de grupos de bolsas financieras con similitudes en su comportamiento bursátil, facilitando su análisis e interpretación.

La metodología para abordar esta investigación es del tipo correlacional, ya que se analiza la relación entre más de dos variables, en particular el comportamiento bursátil de las bolsas financieras que cotizan en Wall Street. La recolección de datos fue tomada de la página oficial de Wall Street, correspondiente al cierre (Acciones negociadas) de 27 bolsas financieras de manera diaria, durante un periodo de cuatro años (2011 a 2014). La base de datos se almacena en el software Excel para su normalización, con el fin de disminuir su variación natural; posteriormente, se aplica el Análisis de Componentes Principales -PCA- en la plataforma Matlab, obteniendo los componentes principales, de donde se tomaran los tres primeros componentes, para determinar la formación de grupos y se mostrarán en un gráfico de dispersión.

En los resultados obtenidos, se destacan el comportamiento particular de las bolsas de valores de Singapur, cuyo mercado de valores ha ido a la baja, y Argentina que ha ido a la alza; ambas bolsas se encuentran muy alejadas de los conjuntos contenedores correspondientes a mercados financieros de otros países.

Palabras clave: PCA, Análisis Multivariado, Análisis financiero bursátil, Análisis técnico financiero.

\begin{abstract}
The objective of this research is to analyze the stock performance of 27 stock markets around the world, using Principal Components Analysis (PCA). This method allows grouping the correlated variables together, obtaining an orthogonal basis where the analysis and interpretation of data is easier.

The data used was collected from the official website of Wall Street every day, at "close trading", from 27 financial markets for the period 2011 - 2014. Then, the database was saved in excel, and after that the PCA method was applied in Matlab software. The Principal components are plotted in an orthogonal basis to represent the formation of groups based on their market performance.

Among the results, the Singapore stock stands out due to its decreasing trend, while the Argentina stock protrudes for its rising trend. Both markets are distant from the container sets from other stock markets.
\end{abstract}

Keywords: Stock Markets, Multivariate Analysis, PCA, Financial Analysis. 


\section{INTRODUCCIÓN}

Las bolsas de valores son entidades que desarrollan su actividad dentro de los mercados de valores y ofrecen oportunidades de negocios. En términos económicos, un mercado financiero es un lugar donde se reúnen aquellas personas interesadas en realizar transacciones con activos financieros, con el objetivo de beneficiar tanto a quienes necesitan dinero para diversos proyectos de inversión como a quien presta ese dinero a cambio de recibir un interés.

Las negociaciones en los mercados bursátiles tienen precios conocidos, los cuales se establecen en tiempo real respetando la normatividad legal de cada país, lo que garantiza seguridad para las acciones que se negocian (Quiroga y Limón, 2011).

En general, las diferentes bolsas de valores en el mundo comparten un funcionamiento similar, en el cual intervienen demandantes de capital, ofertantes de capital e intermediarios (Rueda, 2005).

Cada entidad financiera cuenta con un indicador bursátil, el cual refleja la evolución diaria de las acciones que se cotizan en la bolsa, es decir el indicador reporta si las acciones «fueron a la baja o a la alza».

Predecir el comportamiento bursátil de una bolsa de valores en particular resulta una tarea ardua y por demás difícil. Según Rueda (2005) existen dos enfoques para analizar los mercados financieros:

- El enfoque clásico, el cual consiste en analizar los mercados con base en periódicos o revistas especializados, para así poder predecir el comportamiento bursátil, incluso el analista puede valerse de su intuición para realizar sus predicciones.
- El enfoque técnico, está basado en analizar estadísticamente un fenómeno en particular y mediante técnicas estadísticas y matemáticas intentar predecir su comportamiento en el tiempo.

Si resulta difícil analizar y pronosticar el comportamiento de un indicador de un mercado en particular, es por demás difícil intentar analizar varios indicadores pertenecientes a diferentes mercados financieros interactuando en conjunto.

Dada la problemática planteada, se propone la utilización de una herramienta probada en diversas ciencias, que puede facilitar la interpretación de diversos indicadores, que en este caso son los mercados financieros.

La herramienta propuesta como auxiliar para el análisis técnico es el análisis de componentes principales PCA. Esta herramienta pertenece al análisis multivariado, el cual permite analizar conjuntos de datos de alta dimensionalidad (Tatham, Anderson y Black, 2006).

La limitante principal para el uso de esta técnica es: que los resultados pueden ser malinterpretados si se desconoce el comportamiento de otras variables económicas, es decir, se sugiere complementar esta técnica con el enfoque clásico.

\section{MARCO TEÓRICO}

\section{El mercado de valores}

Según Rueda (2005), el mercado de valores es la base de la economía global, ya que en él se llevan a cabo negociaciones indispensables para mantener viva la economía de los países. En la dinámica bursátil se distinguen dos tipos de mercados, el mercado de capital y el mercado de dinero (Rueda, 2005). 
El mercado de capital, es donde se llevan a cabo transacciones de compraventa de acciones, dichas acciones son parte de un capital social. Cuando una empresa necesita financiarse para diversos proyectos, entonces se colocan acciones en la bolsa de valores, a esta actividad de vender parte del capital para financiar proyectos se le conoce como mercado primario, a todas las transacciones posteriores al mercado primario se le conoce como mercado secundario (Elton, Gruber, Brown y Goetzmann, 2009).

El mercado de dinero es aquel sistema donde se concentran los recursos correspondientes al ahorro de una economía, es decir, ahí se transan ofertas de capital entre personas que cuentan con excedentes monetarios, para prestarlo a demandantes de capital quienes a cambio del préstamo pagaran intereses a los oferentes, los demandantes de capital pueden ser bancos, empresas o gobiernos (Smith, 1997).

La inversión en el mercado de dinero lleva implícito un riesgo, el cual no solo depende del impago sino de las variaciones en la economía, estas variaciones pueden ser cíclicas o intempestivas (Bouchaud y Potters, 2003).

\section{Los indicadores bursátiles}

Los indicadores son un parámetro de la evolución bursátil de las acciones en el mercado accionario, estos índices pueden construirse de diversas maneras, siempre y cuando midan la actividad general de un mercado (Quiroga y Limón, 2011).

Charles H. Dow, en 1884, construyó el índice Dow Jones; para crear el índice contempló el precio de cierre de cada una de las acciones de las once empresas más representativas de los Estados Unidos en ese periodo, finalmente promediaba esos valores y obtenía el indicador.
Para el año de 1896 ya existían dos listas con los indicadores, el de las empresas ferrocarrileras y el de doce empresas industriales, al cual se le concedió el nombre de Dow Jones Industrial Average. En la actualidad existen 4 indicadores del Dow Jones el DJIA Dow Jones Industrial Average, DJTA Dow Jones Transportation Average, DJUA Dow Jones Utility Average y el DJCA Dow Jones Composite Average.

El Dow Jones representa 30 acciones de las 3000 que cotizan en NYSE, lo que apenas representa el $1 \%$, sin embargo en términos monetarios representa $25 \%$ del total del mercado (Rueda, 2005).

\section{Análisis del mercado}

En general, el análisis mediante el enfoque técnico se basa en el estudio de la evolución de los precios de las acciones conforme pasa el tiempo, esta evolución se representa principalmente en gráficas, las cuales sirven de ayuda para decidir cuándo comprar o cuándo vender obteniendo los mayores beneficios (García, 2007).

\section{El análisis multivariado}

Dentro del estudio del análisis multivariado se encuentran las técnicas descriptivas y explicativas. Las técnicas explicativas se usan en la investigación experimental, en donde se consideran simultáneamente un conjunto de variables; por el otro lado, las técnicas descriptivas se usan en contextos no experimentales con el fin de resumir la información que arroja un conjunto de datos.

Dentro de las técnicas explicativas se encuentran las técnicas de regresión lineal múltiple, análisis discriminante lineal, análisis multivariado de varianza y covarianza, análisis de regresión logística y ecuaciones lineales estructurales. 
Por su parte, las técnicas descriptivas contienen a las técnicas de análisis de métodos categóricos, análisis de correlación canónica, análisis de clúster y el análisis de componentes principales (Tatham, et al., 2006).

\section{El Análisis de Componentes Principales -PCA}

Karl Pearson propuso a finales del siglo XIX la técnica de Análisis de Componentes Principales PCA, como una técnica estadística, en particular como parte del análisis de factores, sin embargo debido a la complejidad de los cálculos esta técnica retrasó su avance hasta la aparición de las computadoras (Quiroga y Limón, 2011).

El objetivo de PCA es agrupar las variables que tienen correlación entre sí, separándolas de las que no (Restrepo, Posada y Nogueira, 2012). En el PCA no se interpreta cada uno de los factores, lo que se interpreta es el agrupamiento de las variables (Cuadras, 2014).

Es importante mencionar que al aplicar el análisis de componentes principales PCA, para este caso en particular, no es necesario aplicar las pruebas de Heterocedasticidad, ya que el método considera los componentes más viables de tal forma que las variables agrupadas están altamente correlacionadas entre sí, pero mantienen escasa vinculación a otras componentes (Pena y Sánchez, 2007).

Después de aplicar el PCA a un conjunto de varias variables y diversas observaciones, se obtiene un nuevo espacio reducido, en donde es mucho más fácil el análisis e interpretación de los datos, dicho nuevo espacio, es una combinación lineal de las variables originales.

En el método de análisis de componentes principales, se construye un nuevo sistema de coordenadas con base en los datos originales, donde la varianza mayor será conocida como primer componente principal, de tal forma que el segundo componente será el de mayor varianza solo por debajo del primero y así sucesivamente (Pla, 1986).

El componente principal que arroja mayor información es el primero, posteriormente se va perdiendo información, es decir el segundo componente arroja menos información que el primero y así sucesivamente (Tatham, et al., 2006).

\section{Estado del arte}

Los estudios que se han realizado utilizando técnicas de la estadística avanzada van desde las áreas químicas y biológicas, como es el caso del estudio del Dr. José Luis González Solís, en donde se detecta cáncer de mama usando la espectroscopia Raman y el análisis multivariado, demostrando la efectividad de estas técnicas estadísticas (Martinez-Espinoza, et al., 2008).

Nica en 2011 realizó un análisis de la asignación de recursos financieros dentro de los sistemas de salud utilizando el método de análisis de componentes principales PCA, con base en los resultados de esta investigación se concluye que con un óptimo monitoreo de las variables utilizadas se puede ayudar a combatir las desigualdades en la asignación y utilización de los recursos.

Por su parte, Afanador y Espinoza (2013), con el uso de la técnica de análisis de componentes principales PCA, analizaron tres subsectores de la industria (manufactura, energía y construcción) con la finalidad de encontrar la correlación entre estos diferentes sectores, demostrando que esta técnica puede aplicarse como un auxiliar en la óptima asignación de recursos.

En torno a los temas de desarrollo económico destaca el trabajo de Unceta, Gutiérrez-Goiria, y Goitisolo (2014), en el cual se usó el método de Análisis de Componentes Principales para analizar la relación entre la Asistencia oficial al 
Desarrollo (AOD) y la pobreza en 75 diferentes países durante veinte años.

En el ámbito económico se han realizado trabajos de investigación como el de Díaz, Vermunt y Ramos (2015), en el cual analizan los rendimientos de 21 mercados de valores europeos, obteniendo dos grupos de los mercados de valores, donde cada grupo se caracteriza por diferentes dinámicas que corresponden a diferentes patrones de rentabilidad-riesgo esperado.

En el trabajo realizado por Mellado y Escobari en 2014, se utiliza el modelo de correlación condicional dinámico, propuesto por Engle en 2002, con el objetivo de identificar una correlación positiva entre los mercados financieros de los países de Chile, Colombia y Perú. Su investigación dio por resultado la evidencia de que la creación de la MILA (Mercado Integrado Latinoamericano) aumentó los niveles de correlación dinámica, entre los rendimientos de las acciones.

Por su parte, en el trabajo de Quiroga y Limón (2011) se determinó la correlación existente entre las diferentes bolsas financieras en el mundo usando el PCA y LDA, las cuales son técnicas del análisis multivariado, obteniendo como resultado la formación de grupos, los cuales incluían países cuyos comportamientos bursátiles eran similares. Uno de los resultados interesantes, fue observar un comportamiento característico por parte de Grecia, ya que estaba aislado de los demás conjuntos de países, este comportamiento anómalo se vio justificado debido a que cayó en crisis financiera.

\section{METODOLOGÍA}

Con base en los datos estadísticos de la página de Wall Street, se recopilaron las observaciones diarias correspondientes al cierre (Acciones negociadas) de 27 bolsas financieras, durante un periodo de cuatro años (2011 a 2014); esta base de datos se vaciará en el software Excel, en el cual se procederá a normalizar, con el fin de quitar el ruido a los datos.

El conjunto de observaciones a lo largo de cuatro años formará una matriz de $976 \times 27$, donde cada columna representará el comportamiento bursátil de una bolsa en particular y cada renglón será el volumen de acciones negociadas en un día en específico.

Creada la matriz de datos, se procederá a calcular la matriz de correlación, la cual será de 27x27; luego se calcularán los 27 valores propios con sus respectivos vectores propios, obteniendo así el conjunto de componentes principales, los cuales serán graficados para su posterior análisis e interpretación en la plataforma Matlab.

Para conseguir este nuevo espacio es necesario calcular además de la estadística básica media, varianza y desviación estándar (Lind, Wathen y Marchal, 2012), los siguientes conceptos:

- Coeficiente de correlación, el cual es una medida del grado de asociación lineal entre las variables $x$ e $y$, y su fórmula se representa de la siguiente manera:

$$
\mathrm{r}=\frac{s_{\mathrm{xy}}}{s_{x} s_{y}}(1)
$$

Donde, $s_{x} y s_{y}$ son las desviaciones típicas de las variables "X» e "y» respectivamente, y $s_{x y}$ es la covarianza muestral de "x» e "y», misma que se define como la media de los productos de las desviaciones de "x» e "y», y de sus medias muestrales.

$$
s_{\mathrm{xy}}=\frac{1}{n-1} * \sum_{\mathrm{i}=1}^{n}\left(x_{i}-\bar{x}\right) *\left(y_{i}-\bar{y}\right)(2)
$$

- Matriz de correlación, la cual se obtiene y da por resultado una matriz simétrica, en la cual la diagonal principal es igual a 1.

Esta matriz se puede obtener hallando la matriz de varianza covarianza, para datos que 
se encuentran normalizados (como es este caso).

$$
\begin{gathered}
s_{\mathrm{xy}}=\frac{1}{n-1} * \sum_{\mathrm{i}=1}^{n}\left(x_{i}-\bar{x}\right) *\left(y_{i}-\bar{y}\right)(3) \\
s_{\mathrm{xx}}=\mathrm{s}_{x}^{2} \frac{1}{n-1} * \sum_{\mathrm{i}=1}^{n}\left(x_{i}-\bar{x}\right)^{2}(4)
\end{gathered}
$$

El siguiente paso es calcular los valores propios y vectores propios, correspondientes a la matriz de correlación mediante el uso de la ecuación:

$$
\mathrm{MV}=\lambda V(5)
$$

Donde, $\quad \mathrm{V}=\left(v_{1}, \mathrm{v}_{2}, \mathrm{v}_{3} \ldots v_{n}\right)$, son los eigenvectores, $y\left(\lambda_{1}, \lambda_{1} \ldots, \lambda_{n}\right)$ son los valores propios.

Los valores propios son las raíces de la ecuación:

$$
\operatorname{det}(M-\lambda I)=0,(6)
$$

Donde I es la identidad de M.

Las coordenadas de los vectores propios hallados son los coeficientes de la transformación que hay que realizar para pasar al nuevo espacio de componentes principales (Hernández, 1998).

\section{RESULTADOS Y DISCUSIÓN}

Las bolsas de valores consideradas en el estudio se encuentran representadas y ordenadas alfabéticamente en la tabla 1. Dichas bolsas son las que se encuentran disponibles en la página oficial de Wall Street.

El parámetro que se usó para realizar este estudio fue el indicador de cierre (volumen de acciones negociadas).

Después de recabar las observaciones del cierre de cada una de las bolsas de la Tabla 1, a lo largo de un periodo de 4 años (20112014), se normalizó la matriz de datos con la finalidad de quitar el ruido que impidiera el análisis.

Tabla 1.Bolsas financieras

Table 1.Stock Markets

\begin{tabular}{cl}
\hline 1 & Amsterdam total return general \\
\hline 2 & Argentina MERVAL INDEX \\
\hline 3 & ATX Viena \\
\hline 4 & Australia all ordinaries \\
\hline 5 & Belgium 20 \\
\hline 6 & Bombay Sensex Index \\
\hline 7 & Brasil BOVESPA \\
\hline 8 & Chile IPSA \\
\hline 9 & Financial Times Stock Exchanges \\
\hline 10 & Frankfurt Index Dax \\
\hline 11 & Hong Kong Hang Seng \\
\hline 12 & IPC México \\
\hline 13 & Jakarta Composite Indonesia \\
\hline 14 & Japon NIKKEI225 Index \\
\hline 15 & Kuala Lumpur Composite Malasia \\
\hline 16 & Madrid general \\
\hline 17 & Norway OSE \\
\hline 18 & NYSE New York \\
\hline 19 & París CAC 40 \\
\hline 20 & PSE Composite Filipinas \\
\hline 21 & Seul Composite \\
\hline 22 & Shangai \\
\hline 23 & Singapur Straits Time \\
\hline 24 & Sri Lanka Share \\
\hline 25 & Swiss Market Index \\
\hline 26 & Taiwan \\
\hline 27 & Toronto Composyte \\
\hline Fuente: Elaboración propia con datos de la \\
\hline
\end{tabular}

La gráfica de la Fig. 1, muestra cada una de las 976 observaciones correspondientes a las 27 bolsas financieras a través de cuatro años.

Habiendo normalizado la matriz de datos se procede a calcular los valores propios con sus respectivos vectores propios (27 en este caso), para obtener los componentes principales. 


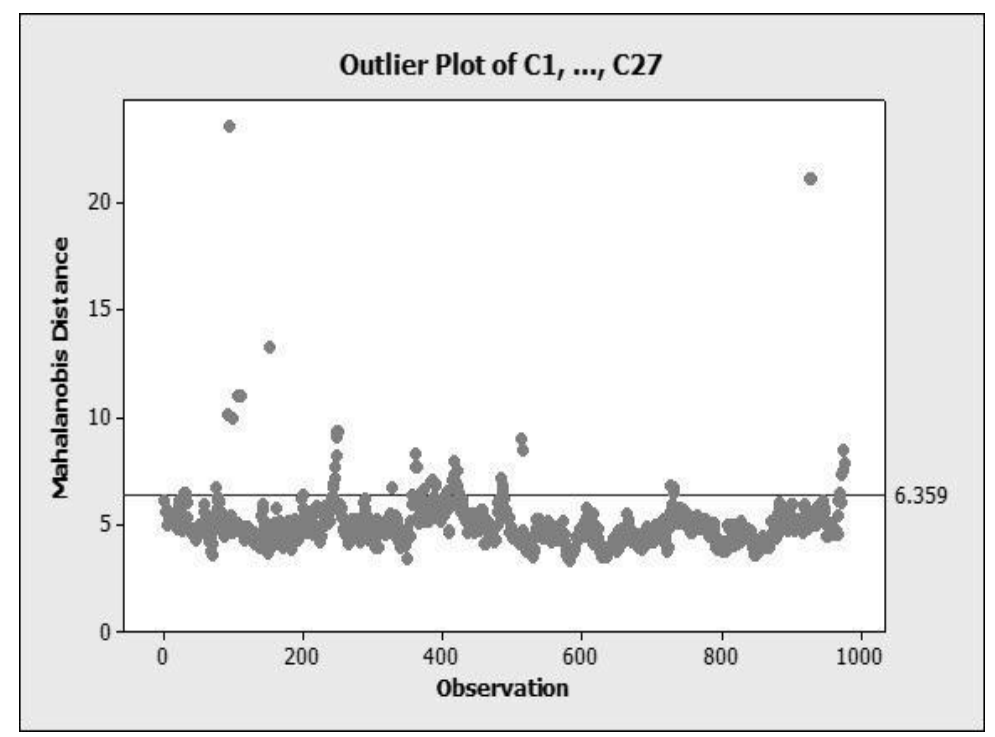

Figura 1. Gráfica de datos normalizados

Figure. 1. Normalize Data Plot Fuente: Elaboración propia

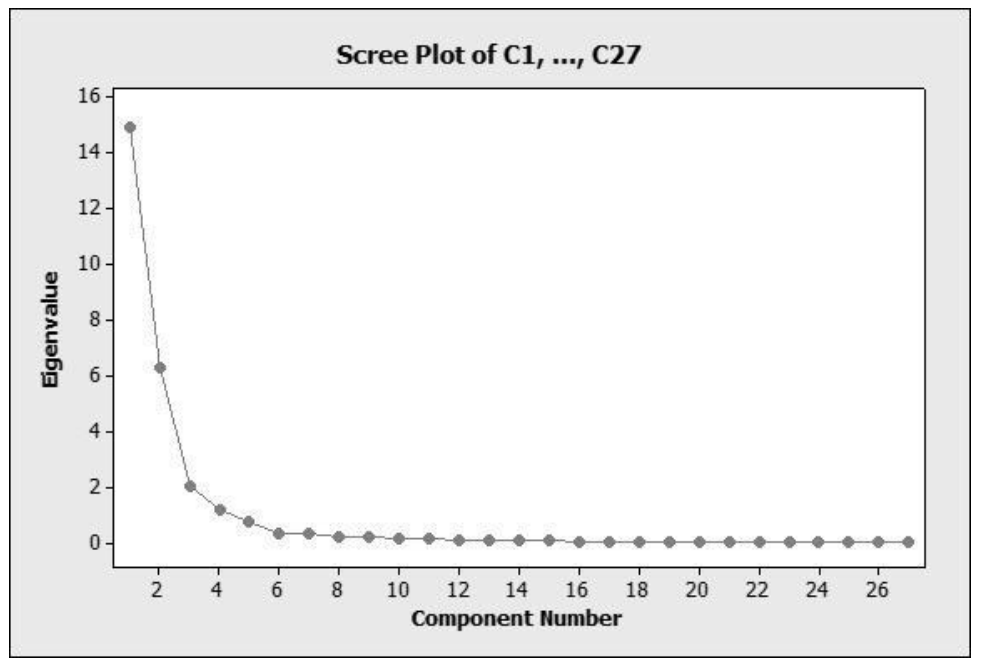

Figura 2. Gráfica de los eigenvalores con respecto de las componentes principales

Figure 2. Graph of the eigenvalues respect Principal Components

Fuente: Elaboración propia

En la Fig. 2, se representa de manera gráfica la varianza explicada por cada uno de los componentes principales, el primer componente explica el $55.2 \%$ de la varianza; el segundo componente explica el $23.3 \%$; y el tercer componente explica el $7.5 \%$ de la varianza, la suma de las ponderaciones da como resultado el $86 \%$, lo cual significa que los primeros tres componentes explican el $86 \%$ de la varianza; en cambio del cuarto componente en adelante es mínima la varianza que se explica.

Cuando se grafican los componentes principales 1 y 2 (Fig. 3), se observa un conjunto contenedor en la parte superior derecha de la gráfica y un conjunto disperso en la parte superior izquierda, al igual que un punto en la parte inferior y otro punto disperso en el lado derecho de la gráfica. 


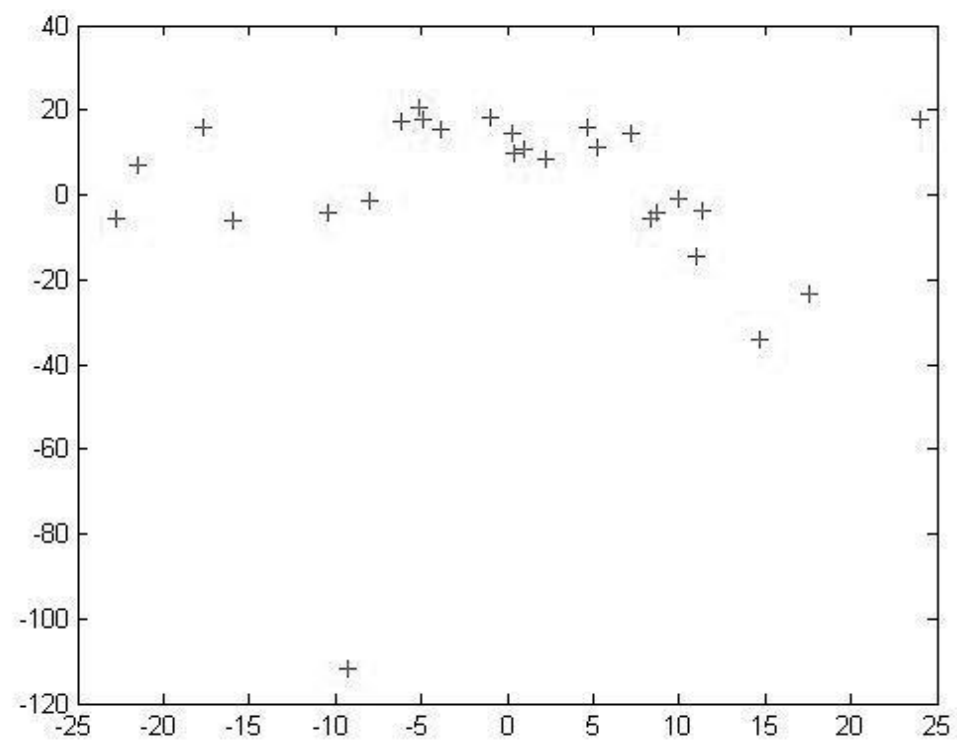

Figura 3. Formación de grupos usando PC1 vs PC2

Figure 3. Formation groups using $P C 1$ vs $P C 2$ Fuente: Elaboración propia

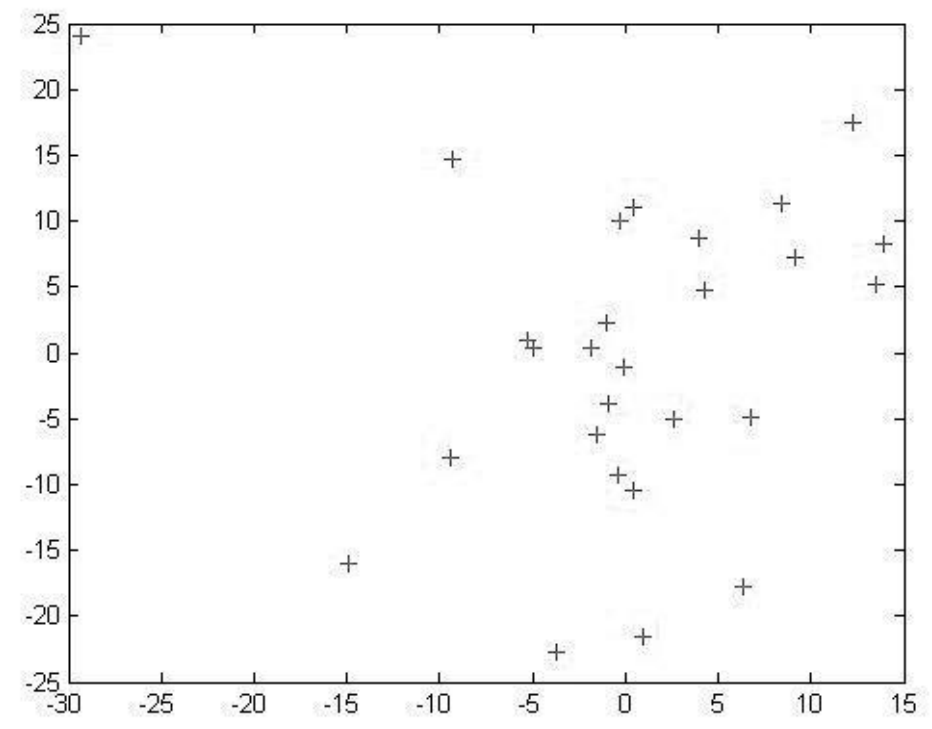

Figura 4. Formación de grupos comparando PC2 vs PC3

Figure 4. Groups Formation comparing PC2 vs PC3

Fuente: Elaboración propia

Al graficar el segundo componente principal con respecto del tercero (Fig. 4), se observa un cúmulo de puntos dispersos dentro de los cuales llama la atención el punto ubicado en la parte superior izquierda.
En la Fig. 5, se observa la gráfica del primer componente con respecto del tercer componente, en la cual se aprecia un conjunto contenedor y dos puntos dispersos, uno en la parte inferior y otro en la parte superior izquierda de la gráfica. 


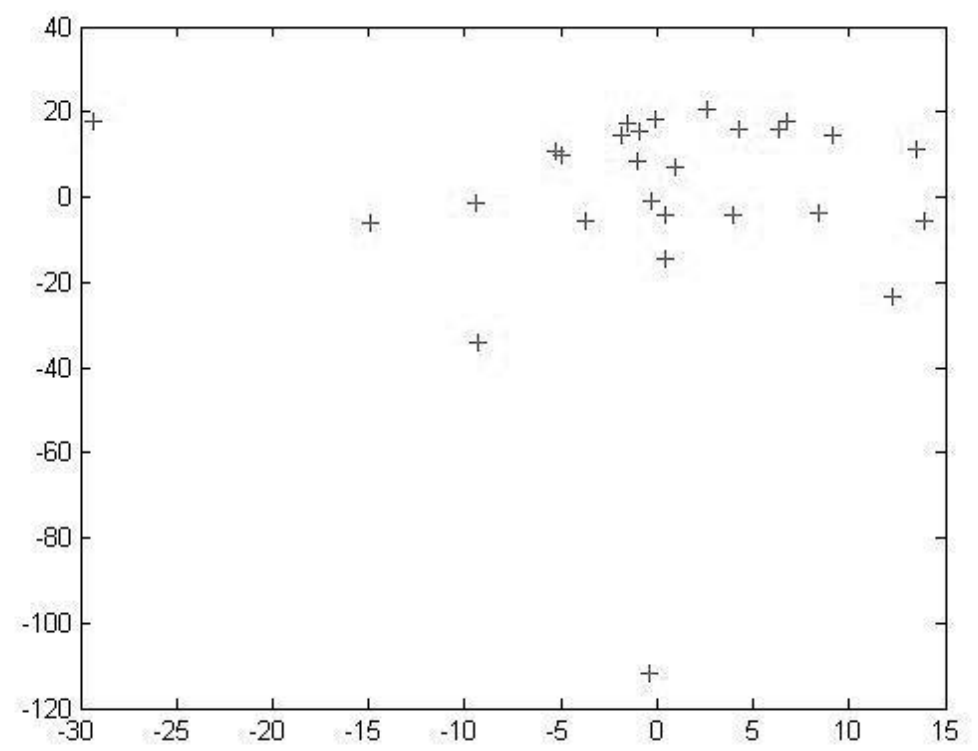

Figura 5. Formación de grupos usando las componentes PC3 vs PC1

Figure 5. Formation of groups using the components $P C 3$ vs $P C 1$ Fuente: Elaboración propia

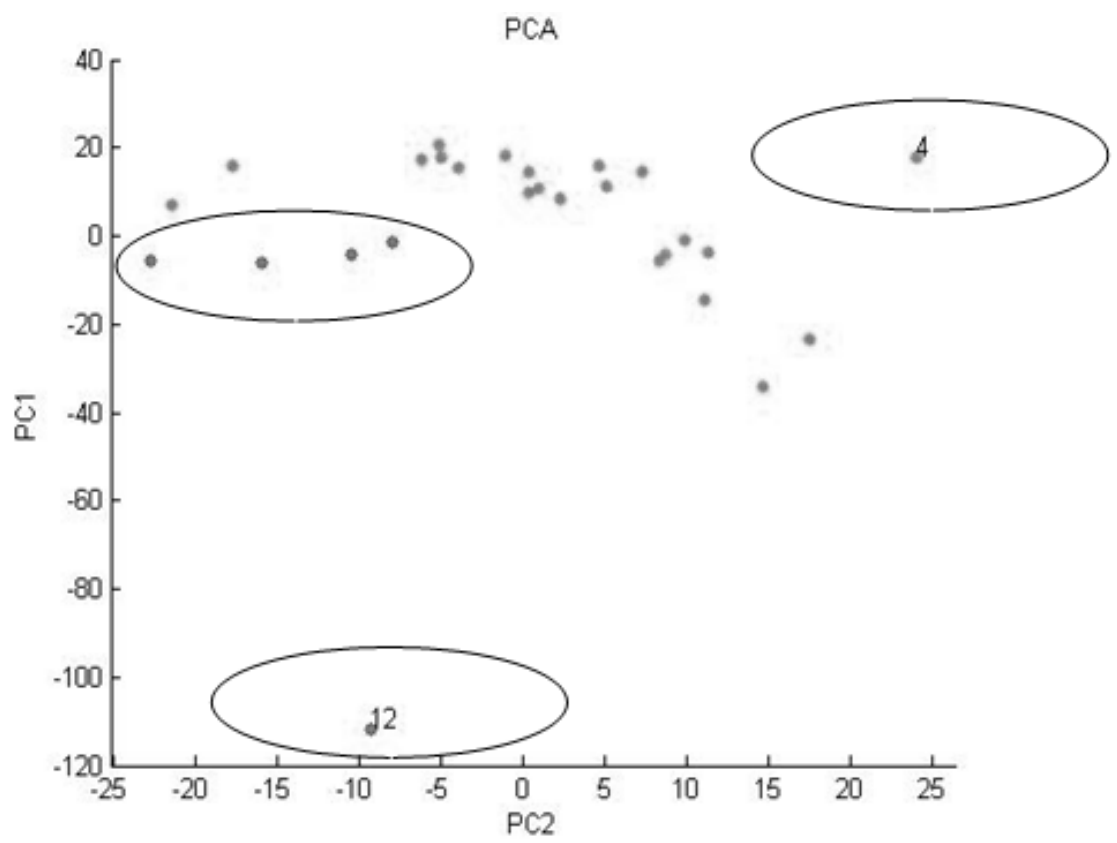

Figura 6. Identificación de Grupos

Figure 6. Cluster's identification Fuente: Elaboración propia

Con base en la información proporcionada por las figuras presentadas, se puede decir que los componentes que mayor información arrojan son los componentes uno y dos.
En la Fig. 6 se observa la identificación de grupos con base en el algoritmo de PCA, en los cuales se distinguen un grupo contenedor que se encuentra fuera de las elipses, el cual 
muestra un comportamiento bursátil similar. También se observa un conjunto disperso, el cual es identificado por la elipse del lado superior izquierdo, este agrupamiento tiene como base su comportamiento bursátil a través del tiempo. Llama la atención el comportamiento particular del punto centrado en la elipse ubicada en la parte inferior de la gráfica; este elemento corresponde a la bolsa de valores de Singapur, por su parte el elemento contenido en la elipse superior derecha mantiene un comportamiento bursátil particular, es por eso que se encuentra alejado de los demás conjuntos con comportamientos similares.

En la Fig. 7, se enumera cada uno de los elementos pertenecientes a cada uno de los grupos quedando ordenados como se muestra en la tabla 2.

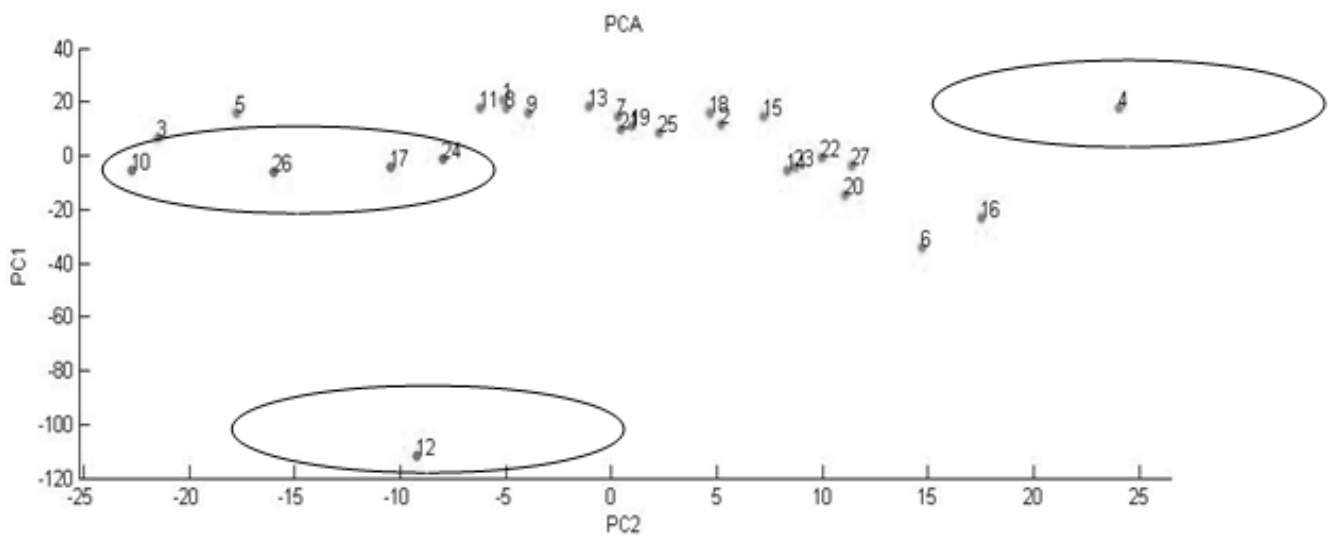

Figura 7. Agrupamiento de Bolsas Financieras

Figure. 7. Grouping Stock markets

Fuente: Elaboración propia

Tabla 2. Agrupamiento de bolsas de valores con comportamiento similar

Table 2.Grouping of stock markets with similar behavior

\begin{tabular}{|c|c|c|c|}
\hline Grupo1 & Grupo2 & Grupo3 & Grupo4 \\
\hline Frankfurt index Dax & Toronto Composyte & Singapur straits time & Argentina MERVAL INDEX \\
\hline Madrid general & IPC México & & \\
\hline Sri Lanka Share & Brasil BOVESPA & & \\
\hline \multirow[t]{18}{*}{ ATX viena } & chile IPSA & & \\
\hline & Japon NIKKEI225 index & & \\
\hline & Australia all ordinaries & & \\
\hline & Seul composite & & \\
\hline & Hong kong hang seng & & \\
\hline & shangai & & \\
\hline & Taiwan & & \\
\hline & Bombay sensex index & & \\
\hline & Jakarta composite indonesia & & \\
\hline & Kuala lumpur composite Malasia & & \\
\hline & PSE composite Filipinas & & \\
\hline & Financial times stock exchanges 100 Londres & & \\
\hline & Paris CAC 40 & & \\
\hline & Belgium 20 & & \\
\hline & Swiss Market Index & & \\
\hline & Norway OSE & & \\
\hline & Amsterdam total return general & & \\
\hline & NYSE New York & & \\
\hline
\end{tabular}

Fuente: Elaboración propia 


\section{CONCLUSIÓN}

En esta investigación se estudió mediante la técnica de análisis de componentes principales PCA el comportamiento bursátil de 27 bolsas financieras pertenecientes a 27 países de todo el mundo, esto con el objetivo de observar su comportamiento. Como resultado del análisis estadístico se observó la formación de grupos con comportamiento similar con base en el indicador de cierre de cada una de las bolsas de valores.

Esto fue posible gracias al análisis de componentes principales PCA, el cual permitió un análisis efectivo de una alta gama de variables y diversas observaciones interactuando en conjunto, obteniendo así una clasificación de bolsas financieras de acuerdo con su comportamiento bursátil observado durante un periodo de 4 años.

Llama la atención el caso de Singapur que en las gráficas se muestra alejado de los demás países. Cuando se observa su comportamiento individual se muestran picos característicos, los cuales indican una debacle en la bolsa de valores de Singapur.

Al analizar individualmente el caso de Argentina, el cual muestra un comportamiento particular en la figura de resultados de $P C A$, se observa que su mercado de valores se ha visto a la alza en los últimos tres años y es por eso que en las gráficas de resultados se observa alejado de los demás conjuntos contenedores.

Esta investigación muestra que el PCA aplicado al estudio bursátil es una poderosa herramienta auxiliar en el análisis financiero, en particular para el enfoque técnico, ya que permite estudiar varias variables al mismo tiempo lo cual complementado con la experiencia empírica (indispensable para hacer interpretaciones del PCA aplicado a los mercados de valores), puede predecir el comportamiento de un mercado en particular y tomar decisiones estratégicas para conseguir los máximos beneficios o en el peor de los casos salir lo mejor librado de una situación adversa.

\section{REFERENCIAS}

Afanador, C. A. y Espinoza, C. D. (2013). PCA: una nueva técnica de investigación de operaciones (Tesis de licenciatura no publicada). Lagos de Moreno, Jalisco, México: Universidad de Guadalajara.

Bouchaud, J. P. y Potters, M. (2003). Theory of financial risk and derivate pricing: from statiscal physics to risk management, Cambridge, Inglaterra: Cambridge University Press.

Cuadras, M. (2014). Nuevos métodos de análisis multivariante. Barcelona, España: CMC Editions.

Diaz, J. G.; Vermunt, J. K. y Ramos, S. (2015). Clustering financial time series: new insights from an extended hidden Markov model. European Journal of Operational Research. 104(3), 382-399.

Elton, E.; Gruber, M.; Brown, S. y Goetzmann, W. (2009). Modern portfolio theory and investment analysis, United States of America: Hamilton printing Company.

Engle, R. (2002). Dynamic condicional correlation: a simple class of multivariate generalized autoregressive condicional heterocedasticity models. Journal of Bussines and Economic Statistics, 20. 339350.

García, A. (2007). Sistema financiero mexicano mercado de derivados. Boca del Río, México.

Hernández, O. (1998). Temas de análisis estadístico multivariado. Costa Rica: Comisión editorial de la Universidad de Costa Rica. 
Lind, D., Wathen, S. y Marchal, W. (2012). Estadística aplicada a los negocios y la economía. México: Mc Graw Hill.

Mantegna, R. N. y Stanley, H. E. (2000). An introduction to econophysics Correlations and Complexity in Finance. Cambridge, Inglaterra: Cambridge University Press.

Martínez-Espinoza, J. C.; González-Solis, J. L.; Frausto-Reyes, C.; Miranda-Beltrán, M. L.; Soria-Fregoso, C.; Medina-Valtierra, J. y Sánchez-Gómez, R. (2008). Raman Spectroscopy: A new Proposal for the Detection of Leukimia Using Blood Samples. AIP Proceedings, 1032, (252).

Mellado, C. y Escobari, D. (2014). Virtual Integration of Financial Markets. A Dynamic Correlation of the Latin American Integrated Market. MPRA, 5(55).

Nica, E. A, (2011). Analysis of alocation of financial resources mangement in health systems in OECD member countries using cluster analysis. Metalurgia International, 16(12), 91-95.

Pena, J. A., Sánchez, J. M. (2007). Disparidades económicas intrarregionales a escala municipal. Evidencia empírica para el caso Gallego. Revista de Estudios Regionales, 81, 15-43.
Pla, L., (1986). Análisis multivariado: método de componentes principales. Washington, DC: Secretaría General de la Organización de los Estados Americanos.

Quiroga, C. A. y Limon, M. U. (2011). Estudio de la correlación entre las diferentes bolsas financieras en el mundo, usando el análisis multivariado (PCA y LDA) (tesis de licenciatura). Universidad de Guadalajara, Lagos de Moreno, Jalisco, México.

Restrepo, M.; Posada, S. y Noguera, R. (2012). Aplication of the principal-component analysis in the evaluation of three grass varieties. Revista Colombiana de Ciencias Pecuarias, (25), 258-266.

Rueda, A. (2005). Para entender la bolsa: financiamiento e inversión en el mercado de valores, México DF, México: Thomson.

Smith, L. F. (1997). Un principiante en Wall Street: Manual para el inversionista no experimentado. México DF, México: SICCO.

Tatham, R.; Anderson, R. y Black, B. (2006). Multivariate Data Analysis, United States of America: Pearson Education.

Unceta, K.; Gutiérrez-Goiria, J. y Goitisolo, L. (2014). Evidencias e interrogantes sobre desarrollo, financiación externa y AOD: un análisis de componentes principales. Revista de Economía Mundial, (36), 153178. 